\title{
Renormalization of operators for excited-state hadrons in lattice QCD
}

\section{Ekaterina Mastropas*}

The College of William and Mary, Williamsburg VA, USA

E-mail: emastropaseemail.wm. edu

\section{David Richards}

Jefferson Lab, Newport News VA, USA

E-mail: dgrajlab.org

\begin{abstract}
One of the primary aims of lattice QCD is to accurately compute the spectrum of hadronic excitations from first principles. However, obtaining an accurate resolution of excited states using methods of lattice QCD is not a trivial problem due to faster decay of excited-states correlation functions in Euclidean space in comparison to those of ground states. To overcome this difficulty, anisotropic lattices with a finer temporal discretization are used.

To go beyond the spectrum, in order to study the properties of the states, one needs to compute corresponding matrix elements. Thus, for example, the quark distribution amplitudes in mesons are given by matrix elements of quark bilinear operators, while in baryons, the corresponding quark distribution amplitudes are related to matrix elements of three-quark operators. To relate the matrix elements calculated on the lattice to those in the continuum, and hence to relate to the measured experimentally, it is necessary to evaluate matching coefficients. In this work we describe the calculation of the matching coefficients using perturbation theory for the improved anisotropic-clover fermion action used for our studies of excited states.
\end{abstract}

Sixth International Conference on Quarks and Nuclear Physics,

April 16-20, 2012

Ecole Polytechnique, Palaiseau, Paris

${ }^{*}$ Speaker. 


\section{Introduction and motivation}

Understanding the hadron spectrum in the light and heavy quark sectors of QCD remains one of the fundamental aims of strong interaction physics. In this ongoing project, we apply methods of perturbation theory to lattice quantum chromodynamics in order to investigate the momentum distribution of quarks inside hadrons, relevant for processes at high momentum transfers. A detailed investigation of the spectrum of mesons composed of light quarks is the goal of Jefferson Lab GlueX experiment at $12 \mathrm{GeV}$, which aims to photoproduce mesons with exotic quantum numbers and reveal the role of gluonic degrees of freedom in the spectrum.

In the case of mesons, their simple valence structure presents the ideal testing ground for our understanding of bound quark systems, and a key aim of this project is to come to a better understanding of the pion form factor, which describes the distribution of charges inside the pion, as well as those of the excited states of the pion and other mesons.

This factor $F_{\pi}\left(Q^{2}\right)$ is defined by

$$
\left\langle\pi\left(p_{f}\right)\left|V_{\mu}(0)\right| \pi\left(p_{i}\right)\right\rangle=F_{\pi}\left(Q^{2}\right)\left(p_{i}+p_{f}\right)_{\mu},
$$

here $p_{i}$ and $p_{f}$ are initial and final momenta, $V_{\mu}$ is vector current, and $Q^{2}$ is the four-momentum transfer. By measuring the pion form-factor at short distances, or high $Q^{2}$, it is possible to study its transition to a regime where quarks dynamics predominates; the $12 \mathrm{GeV}$ upgrade in Jefferson Lab will extend form-factor measurements up to a value of $Q^{2} \approx 6(\mathrm{Gev} / \mathrm{c})^{2}$. The major issue here is that at the largest energy scale where reliable experimental measurements have so far been obtained, the pion form-factor data are far larger than the perturbative QCD asymptotic prediction. Lattice calculations have the potential to address the question of how rapidly the form factor approaches the perturbative-QCD expectations, and whether it is at a scale likely to be accessible to experiment. The corresponding distributions for excited meson states are still largely unexplored in lattice QCD.

The transition from factors to excited baryon states at large $Q^{2}$ are important elements of the CLAS program at $12 \mathrm{GeV}$ [1]. In [2], the first lattice calculations of light-cone distribution amplitudes of the $N^{*}(1535)$ resonance were reported, and these distribution amplitudes were used to calculate the transition form factors to nucleon excited states at large momentum transfers.

The lattice approach to QCD offers a non-perturbative method of performing a computation of the QCD spectrum from first principles. Experiments reveal a rich spectrum of excited states, and lattice calculations can help in identifying their properties. However, obtaining an accurate resolution of excited states using methods of lattice QCD is not a trivial problem due to the faster decay of excited-states correlation functions in Euclidean space in comparison with those of ground states, and the severe degradation in the signal-to-noise ratio at increasing temporal separations. To get a better signal, one needs lattices with a fine lattice spacing in the time direction but, at the same time, it is desirable to avoid finite-volume effects that would be very large were a fine lattice spacing to be used in all four directions given current computational resources. A possible solution to this conflicting requirements is the use of anisotropic lattices with a finer temporal than spatial lattice spacing. The first applications of this approach were to glueballs and to heavy-quark physics. Recent calculations on anisotropic lattices with the use of variational method, which 
employs a large basis of interpolating operators satisfying the symmetries of the lattice, allowed the extraction of the spectrum of excited states, both for mesons [3, 4, 5] and for baryons [6, 7].

In our current research, we are performing perturbative calculations of renormalization factors using an improved anisotropic Sheikholeslami-Wohlert ("clover") action with stout smearing of the gauge links. The main focus in this study is on renormalization of operators from which quark distribution amplitudes can be extracted, i.e., quark bilinear operators in case of mesons, or three-quarks operators for baryons. Lattice perturbation theory provides us with a method for systematically matching regularization schemes so that we could establish the right connection between results obtained within particular lattice scheme and physical continuum theory (or, in other words, between lattice simulations and experimental data).

\section{Calculational details: improved anisotropic lattice action with stout-smeared link variables.}

The action used in our calculations can be represented through the following formulae. For the gauge (gluon) sector we use a $O\left(a^{2}\right)$-improved Symanzik and tadpole improved action [8]:

$$
S_{G}[U]=-\beta\left\{\xi_{0}\left[\frac{4}{3} \sum_{x, s} P_{t s}-\frac{1}{12} \sum_{x, s} R_{t s}\right]+\frac{1}{\xi_{0}}\left[\frac{5}{3} \sum_{x, s<s^{\prime}} P_{s s^{\prime}}-\frac{1}{12} \sum_{x, s<s^{\prime}}\left(R_{s s^{\prime}}+R_{s^{\prime} s}\right)\right]\right\}
$$

Here $\beta=\frac{2 N_{c}}{g_{0}^{2}}$ ( $g_{0}$ is a coupling constant); $\xi_{0}$ denotes a bare anisotropy parameter. $P_{s s^{\prime}}$ is a plaquette, and $R_{s s^{\prime}}$ is a $2 \times 1$ rectangular Wilson loop, with two links long in the $s^{\prime}$ direction, summed over all lattice sites.

For the fermion action, we use the "clover" action with stout-smearing of gauge links in the spatial directions. This fermion sector can be represented as:

$$
\begin{aligned}
S_{F}[\psi, \bar{\psi}, U]= & a_{t} a_{s}^{3} \sum_{x} \bar{\psi}(x)\left\{m_{0}+\gamma_{t} \nabla_{t}-\frac{a_{t}}{2} \Delta_{t}+v_{s} \sum_{k}\left(\gamma_{k} \nabla_{k}-\frac{a_{s}}{2} \Delta_{k}\right)+\right. \\
& \left.+\frac{1}{2}\left[c_{t} a_{s} \sum_{k} \sigma_{t k} F_{t k}+c_{s} a_{s} \sum_{k<l} \sigma_{k l} F_{k l}\right]\right\} \psi(x) .
\end{aligned}
$$

Here $a_{t}$ and $a_{s}$ are temporal and spatial lattice spacings respectively, $\nabla_{k}$ and $\Delta_{k}$ are the covariant derivatives and Laplacian corresponding to the Wilson term, respectively, and $F_{k l}$ is a clover-leaf discretized field strength tensor. The clover coefficients $c_{t}$ and $c_{s}$ must to be tuned; their tree-level values for massless quarks are $c_{t}=\frac{1}{2}\left(v_{s}+\frac{1}{\xi_{0}}\right)$ and $c_{s}=v_{s}$. Setting the coefficient $v_{t}$, multiplying the kinetic term, to $v_{t}=1$, measured aspect ratio $\frac{a_{s}}{a_{t}}$ specifies the renormalized gauge anisotropy.

To smooth the short-distance fluctuations in the clover fermion action, we use three-dimensional stout-smeared links. Following the analytic algorithm of smearing link variables, which is presented in [9], at each step $n$ the links $U_{\mu}^{(n)}(x)$ are mapped into links $U_{\mu}^{(n+1)}(x)$ :

$$
U_{\mu}^{(n+1)}(x)=\exp \left(i Q_{\mu}^{(n)}(x)\right) U_{\mu}^{(n)}(x),
$$

where the matrix $Q_{\mu}(x)$ defined as a product of weighted sum of the perpendicular staples which begin at lattice site $x$ and terminate at neighboring site $x+\mu$, and $U_{\mu}^{+}(x)$. After iterating this step $n_{\rho}$ times (twice in our case), we can finally produce so-called "stout link" $\tilde{U}$ :

$$
U \rightarrow U^{(1)} \rightarrow U^{(2)} \rightarrow \ldots \rightarrow U^{\left(n_{\rho}\right)} \equiv \tilde{U} .
$$


We use the common choice of staple weights,

$$
\begin{aligned}
& \rho_{i j}=\rho, \\
& \rho_{4 \mu}=\rho_{\mu 4}=0,
\end{aligned}
$$

so only the spatial links are smeared. In particular, our smearing parameters are $\rho=0.22$ and $n_{\rho}=2$.

\section{Feynman rules on the lattice: first results}

Due to the breaking of Lorentz symmetry, lattice perturbation theory in general is more complicated than continuum perturbation theory. But the process of deriving Feynman rules on the lattice is essentially the same as in continuum theory: one firstly needs to perform a Taylor expansions of the particular lattice action in order to obtain the expressions for propagators and vertices, and then it is possible to build Feynman diagrams from these rules and evaluate them by integrating over phase space. This common procedure is explained in great details in [10]. Explicitly, we define Fourier transforms on the lattice; in infinite volume (a standard setting of perturbation theory) they are given by formulae:

$$
\begin{gathered}
\psi(x)=\int_{-\frac{\pi}{a}}^{\frac{\pi}{a}} \frac{d^{4} p}{(2 \pi)^{4}} e^{i x p} \psi(p), \\
\bar{\psi}(x)=\int_{-\frac{\pi}{a}}^{\frac{\pi}{a}} \frac{d^{4} p}{(2 \pi)^{4}} e^{-i x p} \bar{\psi}(p), \\
A_{\mu}(x)=\int_{-\frac{\pi}{a}}^{\frac{\pi}{a}} \frac{d^{4} k}{(2 \pi)^{4}} e^{i\left(x+a \frac{\mu}{2}\right) k} A_{\mu}(k),
\end{gathered}
$$

Then, by substituting these Fourier transforms into terms of the action, corresponding to quark and gluon propagators or quark-gluon vertices, we can get expressions for Feynman rules. For the anisotropic clover action, we find the following:

- for quark propagator:

$$
S_{\bar{q} q} \sim\left[m_{0}+\frac{i}{a_{t}} \gamma_{t} \sin \left(a_{t} p_{t}\right)+\frac{2}{a_{t}} \sin ^{2}\left(\frac{a_{t} p_{t}}{2}\right)+\frac{i}{a_{s}} v_{s} \sum_{s} \gamma_{s} \sin \left(a_{s} p_{s}\right)+\frac{2}{a_{s}} v_{s} \sum_{s} \sin ^{2}\left(\frac{a_{s} p_{s}}{2}\right)\right]^{-1},
$$

- for quark-gluon vertex:

$$
\begin{aligned}
S_{\bar{q} g q} & \sim g_{0}\left[\sin \left(\frac{a_{t}\left(p+p^{\prime}\right)_{t}}{2}\right)+i \gamma_{t} \cos \left(\frac{a_{t}\left(p+p^{\prime}\right)_{t}}{2}\right)+\right. \\
& +v_{s} \sum_{s} \sin \left(\frac{a_{s}\left(p+p^{\prime}\right)_{s}}{2}\right)+i v_{s} \sum_{s} \gamma_{s} \cos \left(\frac{a_{s}\left(p+p^{\prime}\right)_{s}}{2}\right)+ \\
& +\frac{c_{t}}{a_{t}} \sum_{s} \frac{\sigma_{t s}}{2}\left\{a_{t} \sin \left(a_{s} k_{s}\right) \cos \left(\frac{a_{t} k_{t}}{2}\right)-a_{s} \sin \left(a_{t} k_{t}\right) \cos \left(\frac{a_{s} k_{s}}{2}\right)\right\}+ \\
& \left.+\frac{c_{s}}{a_{s}} \sum_{s<s^{\prime}} \frac{\sigma_{s s^{\prime}}}{2}\left\{a_{s} \sin \left(a_{s^{\prime}} k_{s^{\prime}}\right) \cos \left(\frac{a_{s} k_{s}}{2}\right)-a_{s^{\prime}} \sin \left(a_{s} k_{s}\right) \cos \left(\frac{a_{s^{\prime}} k_{s^{\prime}}}{2}\right)\right\}\right] .
\end{aligned}
$$

Here $p$ and $p^{\prime}$ are the quark momenta, and $k$ is the gluon momentum. 


\section{Current state and future plans}

While controlling short-distance fluctuations, stout-smeared links used in our study complicate significantly the process of calculation. Once we have derived the Feynman rules for the stout-link action, we are going to apply these rules for evaluation of Feynman diagrams corresponding to quark bilinear operators and thereby calculate matching coefficients in order to confront experimental data.

We are also beginning the computation of the low moments of the meson distribution amplitudes for both the ground and excited states using the anisotropic lattices employed in the study of the excited meson spectra [5]. When combined with the perturbative program outlined here, this will enable us to go beyond the spectrum to explore the properties and internal structure of these states.

\section{Acknowledgments}

We are grateful for useful discussions with Christopher Monahan and Justin Foley. We acknowledge support from U.S. Department of Energy contract DE-AC05- 06OR23177, under which Jefferson Science Associates, LLC, manages and operates Jefferson Laboratory.

\section{References}

[1] I. Aznauryan et al, Theory Support for the Excited Baryon Program at the Jlab 12 GeV Upgrade, [nucl-th/0907.1901v3].

[2] V. Braun et al, Electroproduction of the N(1535) resonance at large momentum transfer, Phys. Rev. Lett. 103 (072001) 2009 [hep-ph/ 0902 . $3087 \mathrm{v} 1]$.

[3] J. Dudek et al, Highly excited and exotic meson spectrum from dynamical lattice QCD, Phys. Rev. Lett. 103 (262001) 2009 [hep-ph/ 0909.0200 v2].

[4] J. Dudek et al, Toward the excited meson spectrum of dynamical QCD, Phys. Rev. D 82 (034508) 2010 [hep-ph/1004.4930v1].

[5] J. Dudek et al, Isoscalar meson spectroscopy from lattice QCD, Phys. Rev. D 83 (11502) 2011 [hep-lat/1102.4299v1].

[6] R. Edwards et al, Excited state baryon spectroscopy from lattice QCD, Phys. Rev. D 84 (074508) 2011 [hep-ph/1104.5152v2].

[7] J. Dudek, R. Edwards. Hybrid Baryons in QCD, [hep-ph/1201.2349v1].

[8] J. Foley, C. Morningstar, Tuning improved anisotropic actions in lattice perturbation theory, in proceedings of Lattice-2008 conference POS (LATTICE2008) 212, [hep-lat/0810.4477v1].

[9] C. Morningstar, M. Peardon, Analytic smearing of SU(3) link variables in lattice QCD, Phys. Rev. D 69 (054501) 2004 [hep-lat/0311018v1].

[10] S. Capitani, Lattice perturbation theory, Physics Reports 382 (113-302) 2003 [hep-lat/0211036v2]. 